\title{
Research on adding print file layout and layout rule
}

\author{
Li Jiangchun \\ Hubei University of Police, Wuhan 430035, China \\ lijiangchun@126.com
}

Keywords: Adding and altering documents; layout rules; document inspection

\begin{abstract}
In the process of adding printed and altered documents, the margins, line spacing, word spacing, and parallelism of printed documents are usually the main points of inspection. However, how to change the layout features during the process of adding and modifying needs further discussion. This article in the exclusion of human factors and mechanical fault conditions, a large collection of 868 experimental samples, using the method of measurement and statistics, explore the editing software version version of the Windows operating system in the condition of different characters, using the same document typesetting, printing documents add change layout word spacing, line spacing parallel, law and so on. Setting does not affect the document word space and line space, different systems and different text editing software, add print alter documents will show in violation of law phenomenon in parallel layout rules, may also appear uneven margins phenomenon paragraph indent neat, this is our test to identify whether there is an important basis to add print the.
\end{abstract}

\section{Introduction}

\subsection{Altered files Research Status}

Altering documents refers to the false documents made by using various forgeries to alter the original content or original appearance of the original document on the basis of the original document. Tim change is one of the common means. With the changes made Tim handwritten documents of arbitrariness and convenience is different, the print file Tim Gerze more difficult and demanding, and it is often more performance for the "add", "change" process is due to file for printing production is not convenient to change and extremely rare, so the research of the paper relates only to the altered document "Tim" which alters the way, so called "add print alter documents".

As everyone knows, the document examination practice, often encounter all kinds of printing document evidence, the domestic and foreign research more dependent or focus on the injection machine in use, produced in the process of maintenance marks, at the same time it has been reported that the use of toner / water analysis in identification of printer types of literature, therefore, to determine whether there is to add the fact that altered document evidence, mainly carries on the analysis from the documents distribution, morphological characteristics, ink ink composition etc., the direct observation method, comparison method, micro image processing software, component analysis and other methods of inspection. In recent years, with the in-depth study of the print file to print on the gear mark, color laser printing ink markings and satellite tracking code, print mode, printing ink step characteristics, inherent characteristics of the fringe, and explores the test technology and provide new method for adding print altered document inspection identification, which will analysis and judgment of the characteristics of layout of observation, layout of law is a feature to identify altered paper is very important to add printing.

But we also see the exposition on the layout regularity of the previous study focused on human factors and mechanical printer hardware differences, and differences of software will result in added change of print layout of falsified documents, the regularity of change has not yet grasp, based on this, this study focused on the editing software version in the condition of different versions of the Windows operating system and different characters, explore the layout rules add print files which 
have no change, in order to reveal the fact of the existence of altered add.

\subsection{Ask Questions}

No matter what machine is used in the suspect part and what method is added or altered, it will need to be edited by the document editing software on the previous computer, and then printed on two or more times on the basis of the original file. So we set in human factors and hardware mechanical failure under the same conditions, the difference will cause software add printing altered file, if it is, this difference is derived from different versions of the same version of form, or differences in their formation, is also formed by chance is inevitable, and whether the inspection and appraisal of value? All of these need our further exploration.

\section{Materials and Methods}

\subsection{Experimental Equipment}

Ruler, laser printer, SANSUNG M2070 and Toshiba 220s, Epson C65 scanner, windows system computer and Photoshop 7 software.

The computer operating system selects 3 were Windows XP, Windows 7, Windows 10, the following 5 kinds of document editing software: Microsoft Word2003, Microsoft Word2007, Microsoft Word2010, Microsoft Word2013 and WPS Office. In addition to the Windows XP, which does not support Microsoft, Word2013, there are 14 combinations of computer operating systems and document editing software.

\subsection{Sample Making Method}

Because this experiment only studies the computer operating system and the document editing software at the same time, adds, prints, changes the document in the layout, the layout rule difference, therefore only designs the same machine to add, to print, to change the document sample. Print sample documents, unified page set to the upper and lower margin, $23 \mathrm{~mm}$, about $24 \mathrm{~mm}$ margin, row spacing is set to single line spacing.

Sample files for each combination in three ways: without printing, add bibliographic (i.e. adding print content in a text at the end), paragraph (i.e. add paragraphs in print with a section of text) were set up, and in accordance with the five number (No. five, No. four, fourth mistress, No. No. three) and three fonts (Arial, italics, bold) print, each combination can collect samples 31. Add print, first to add text to white, print, and then add text to black, other words change white word, two print, that is to complete the add text. Two laser printers are printed in each normal mode, and 868 samples are collected by 14 software systems.

\subsection{Measurement Method}

Measurement of word spacing: 15 consecutive character of a line in the document paragraph coherence parameter, measuring the length of the number of characters divided by 15 word spacing, add class only add some words for measuring the first word spacing in the measurement of the.

Measuring the spacing of selected rows in the same paragraph parameters of the document, measure the top line from the first to the last line of the top of the baseline baseline distance, and then divided by the number of rows in the distance from line spacing, line spacing is measured only add class add some words in the first row after measurement.

\section{Results and Analysis}

\subsection{Layout Rule Without Status}

Without adding samples measuring two kinds of printer 14 software system combination, found in the document layout under the same (No. four font, single spaced, computer operating system different text editing software, does not affect the sample document word spacing and line spacing, 
all row spacing is $0.493 \mathrm{~cm}$, word spacing are $1.1 \mathrm{~cm}$.

\subsection{Add Status Layout Rules}

\subsubsection{Line spacing and word spacing change law with the Same group software system}

Select two printer with a group of software systems (Windows 10 and Word 2007 operating system software version) based on the contents of the original document on the form were added to print different font when the sample, add sample word spacing and line spacing of segments were measured after adding text.

We found the following phenomena: first, no papers after adding or after paragraph to add, five word spacing is $0.37 \mathrm{~cm}$. Two, the add class, five words and small four words add part of the first row spacing is $0.8 \mathrm{~cm}$, less than four of the original font spacing $1.1 \mathrm{~cm}$, add class four font for the phenomenon of spacing is 0.55 greater than the period after. In the end of three, add class, word spacing $0.423 \mathrm{~cm}$ four words less than four original font character spacing $0.493 \mathrm{~cm}$, add a class four font character spacing is less than $0.426 \mathrm{~cm}$ at the same time section. In the end of four, add class, word spacing is greater than the original word, mistress $0.526 \mathrm{~cm}$ No. four font character spacing $0.493 \mathrm{~cm}$, add class mistress font character spacing section is less than $0.533 \mathrm{~cm}$ at the same time. After adding some mistress font spacing unchanged, and after adding class mistress font spacing is the same as $1.1 \mathrm{~cm}$. No. five, No. three mistress bold and bold in text add, word spacing is slightly larger than the mistress and the mistress of the font Arial word spacing, and still less than the period after adding on word spacing 0.533 mistress, from this name began to show effects of font word spacing. Six, different brands of laser printers, under normal circumstances will not affect the word spacing and line spacing.

\subsubsection{Line spacing and word spacing change with the Different groups of software systems}

We were measured in different groups of software system after add and add text sample word spacing and line spacing, such as No. five word spacing is $0.370 \mathrm{~cm}$, the spacing is $0.8 \mathrm{~cm}$, fourth, word spacing is $0.423 \mathrm{~cm}$, the spacing is $0.8 \mathrm{~cm}$, the addition of state, using the same document editing and typesetting format, different system version and different document editing software version of the print sample words and row spacing were not change.

\subsubsection{Adds parallel features to altered text and paragraph indent features}

Through the experiment we found that when the same machine add print, due to the two feed, paper placed in the feeder device will appear deviation, will violate the parallel phenomenon, which is mainly characterized in: add content words and original sample lines are not in the same horizontal line; add content word the line will appear after the uneven margins indented homogeneous phenomenon.

Rule of layout layout when 3.3 characters change

Through experiments, we know that when the font size is changed, the word spacing and the space between lines vary. When the size adjustment becomes large, if from four font font adjustment as the mistress, word spacing, line spacing shortened when adjusted for the size unchanged; between three to a smaller size, selected character lines where the axis will overall downward, the other word line spacing unchanged; when adjusted to a font size. The four font spacing to accommodate a number of words, the character of the word line spacing will increase, the other word line spacing unchanged; when the size adjustment of less than four words, the word for word the character spacing will be reduced along with the other words, the spacing of the same.

\section{Conclusion}

So we can draw the following conclusions: 1 , in the same document layout, whether or not to add add print to print the document, in different systems and different text editing software to set the same layout, will not affect the sample document word spacing and line spacing. 2 , if the current setting for 
single spaced paragraph spacing, brand value is less than 0.5 times per pound of row spacing, line spacing of 0.5 times the unit spacing; if the size is between $0.5-1$ pounds value times the row spacing, line spacing of 1 times the unit spacing; if the size value at 1-1.5 pounds spacing between the line spacing 1.5 times the unit spacing. 3 , add, print, change files, in the layout of the layout of the law will show a violation of the parallel nature of the phenomenon, there may be uneven margins of the page paragraph indentation. This is because if the text font size larger than the original file name, add the first line to keep the word axis parallel with the original document, the original words must be added if the axis moves; text size smaller than the original file name, add the first line to keep the word axis parallel with the original document, add the word line axis must be down. Therefore, adding part of the line spacing is bound to violate the rules of line spacing of the original file, and can be used as the basis for inspection and identification.

\section{Acknowledgements}

Project supported by the key scientific research project of Hubei Provincial Education Department, 2016: Research on methods of adding, printing, altering, and identifying documents, D20164201

\section{References}

[1] Chang Jianping, Tan Jianwei, Yang Xiaodong. Examination of computer printed, altered, forged documents [J]. police technology, 2001 (4): 36-39.

[2] Wang Lizhi. Verification of computer printing, alteration and forgery documents [J]. Journal of Liaoning Police College, 2005 (5): 65-66.

[3] Liu Jianhua, Zhang Feng. Application of print features in identification of altered documents [J]. criminal technology,.2010 (4).44-47.

[4] Tan Jianwei, Yang Xiaodong. Experimental research on Printing additional text content inspection [J]. Journal of Public Security University (NATURAL SCIENCE EDITION),.2001 (3).12-17.

[5] Jia Yuwen, Zou Mingli. Chinese criminal technology Daquan document inspection volume [M]. Beijing: Chinese People's Public Security University press, 2002.

[6] Huo. On the laser printer Jingjing add [J]. inspection law and social changing file (early), 2014 (2): 188, 195.

[7] Li Jia. Analysis of adding printed documents. [J]. rule of law and Economics (late), 2013 (4): 126-127.

[8] Zhuang Lin, Pan Guangcheng. Using Photoshop software to check additional printed documents [J]. Journal of Hunan Public Security College, 2010 (5): 117-120.

[9] Qiang Xiaoying, Shi Shaopei. With the machine add printing inspection technology research [J]. Chinese judicial identification, 2015 (1): 55-58.

[10] al. Test method of adding print content changing file [J]. police inquiry technology, 2010 (6): 41-42.

[11] Tan Jianwei, Yang Xiaodong. Experimental research on Printing additional text content inspection [J]. Journal of Beijing University of public safety (NATURAL SCIENCE EDITION), 2001 (3): 12-17.

[12] Wang Yue, Zhang Haihui, Wang Yong. New method of adding printed document inspection [M]. China judicial expert, 2008.

[13] Wang Jinsheng. Verification of computer printed document change cases [J]. Journal of Beijing University of public security, 1998 (1): 86-87. 
[14] Wang Lizhi. Verification of computer printing, alteration and forgery documents [J]. Journal of Liaoning Police College, 2005 (5): 65-66.

[15] Lin Hong, Lin Liping, Zhang Lei. Identification of printed documents by addition method revision. Journal of Chinese People's Public Security University (NATURAL SCIENCE EDITION), 2012 (2): 22-25.

[16] Lin Zhuang. A discussion on the print file to append text content to test [J]. Journal of Guangzhou City Public Security Management Institute, 2008 (4): 25-28. 